\title{
Osteogenesis of adipose-derived stem cells from patients with glucose metabolism disorders
}

\author{
Aleksandra Skubis-Sikora ${ }^{1} \mathbb{D}$, Bartosz Sikora ${ }^{*^{*}}$ D, Agnieszka Witkowska ${ }^{2}$, Urszula Mazurek ${ }^{3}$ and Joanna Gola ${ }^{4}$ (D)
}

\begin{abstract}
Background: Adipose derived stem cells (ADSCS) are clinically widely used somatic stem cells obtained from white adipose tissue. They are characterized by ability to differentiate e.g. into osteoblasts and might successfully regenerate bone tissue in fracture repair. However, the main problem of somatic stem cells is a documented influence of various diseases, drugs or age which can inhibit cells activity. Therefore, in the present study, we investigated the influence of insulin resistance (IR) and type 2 diabetes (T2D) on the proliferation and differentiation potential of ADSCS.

Methods: The fat from subcutaneous abdominal adipose tissue was acquired by lipoaspiration from 23 voluntary participants, divided into three groups: with diabetes type 2, with insulin resistance and control healthy donors. The proliferative potential was analyzed by cell cytotoxicity assays and by mRNA expression of genes connected with proliferation. Flow cytometry was done for identifying proteins characteristic for mesenchymal stem cells and an analysis of osteogenic differentiation potential based on the assessment of osteogenic markers by real time RTqPCR, and the evaluation of calcium deposition were also performed.
\end{abstract}

Results: The results showed that diabetes type 2 lowered the activity of ADSCs in proliferation assays and changed their phenotypical characteristics. Interestingly, we observed differences in the proliferation potential of ADSCs in patients with insulin resistance, which is often the first phase of diabetes, compared to the control. It might suggest that insulin resistance, early-stage T2D, alters the activity of cells. Moreover, expression of osteogenesis markers was higher in cells from T2D patients than in cells from patients with IR and control.

Conclusion: We conclude that type 2 diabetes changes the activity of stem cells, and insulin resistance influences on the proliferation of ADSCS.

Keywords: Metformin, Mesenchymal stem cells, Metabolic impairment, type 2 diabetes, insulin resistance

\section{Introduction}

Diabetes mellitus is a chronic metabolic disease that is caused by abnormalities in insulin secretion and by disorders in the hormone signaling pathway (Jiao et al. 2015). Type 1 diabetes (T1D), called insulin-dependent diabetes, is characterized by the loss of the ability of beta

\footnotetext{
* Correspondence: bsikora@sum.edu.pl

${ }^{1}$ Department of Cytophysiology, Chair of Histology and Embryology, Faculty of Medical Sciences in Katowice, Medical University of Silesia in Katowice, ul. Medyków 18, C2/108, 40-752 Katowice, Poland

Full list of author information is available at the end of the article
}

cells to produce insulin. The basis for this phenomenon is an autoimmune process that destroys beta cells either by apoptosis or necrosis (Stankov et al. 2013). Type 2 diabetes (T2D), known as non-insulin-dependent diabetes, accounts for $90-95 \%$ of all diabetes cases, and its main cause is insulin resistance (IR) (Simpson et al. 2015). If functioning properly, beta cells secrete an optimum amount of insulin, but this amount is not sufficient to compensate for the resistance of tissues to the hormone. Factors predisposing for this condition are obesity, physical inactivity, and age. T2D usually occurs

(c) The Author(s). 2020 Open Access This article is licensed under a Creative Commons Attribution 4.0 International License, which permits use, sharing, adaptation, distribution and reproduction in any medium or format, as long as you give appropriate credit to the original author(s) and the source, provide a link to the Creative Commons licence, and indicate if changes were made. The images or other third party material in this article are included in the article's Creative Commons licence, unless indicated otherwise in a credit line to the material. If material is not included in the article's Creative Commons licence and your intended use is not permitted by statutory regulation or exceeds the permitted use, you will need to obtain permission directly from the copyright holder. To view a copy of this licence, visit http://creativecommons.org/licenses/by/4.0/. 
in adults, often in the elderly (Simpson et al. 2015). Effective therapy for this type of diabetes is physical activity (O'Hagan et al. 2013), a proper diet (Evert et al. 2013), and pharmacotherapy (Amin and Suksomboon 2014).

The impact of diabetes on bones formation is very complex. Osteogenesis is adversely affected by abnormalities in the insulin signaling pathway (Evert et al. 2013). Activation of insulin receptors in osteoblasts stimulates their proliferation and induces the synthesis of collagen and osteocalcin. Osteocalcin in turn stimulates beta-cell proliferation and the secretion of insulin as well as increases testosterone production by the Leydig cells. The abnormal activity of insulin therefore leads to a reduction in osteocalcin production, which exacerbates insulin deficiency and leads to decreased testosterone production. Both osteocalcin and testosterone are osteogenic factors, and their reduced production negatively affects bone mass and the process of bone remodeling, which increases the risk of fractures (Yan and Li 2013).

Mesenchymal stem cells (MSCs) are somatic, multipotent cells that can be derived from different tissues. One of the richest sources of MSCs is adipose tissue (adiposederived stem cells [ADSCs]). There are many experiments that show the advantages of using MSCs in regenerative medicine (Dzhoyashvili et al. 2014), though some studies suggest that abnormal insulin activity negatively affects MSCs, causing the loss of their proliferative potential and weakening their differentiation potential into osteoblasts (Yan and Li 2013). However, most of the research focus on experiments with animal models or on standardized cells from cell banks in vitro. These experiments only create a condition similar to human organism and fully studying complex interaction is not possible.

Therefore, the aim of the study was to analyze the activity of ADSCs from patients who did not receive any treatment and had insulin resistance, which is a precursor stage to type 2 diabetes, and also cells from patients with T2D in comparison to non-diabetic patients.

\section{Material and methods \\ Patients}

The fat from subcutaneous abdominal adipose tissue was acquired by lipoaspiration from 23 participants. All participants were divided into three groups: T2D group with 9 patients ( 3 men, 6 women, average age 45,3 years, average time of diabetes 6,2 years, all of them took metformin in highest tolerable doses, average dose $1,5 \mathrm{~g} / \mathrm{d}$ ), IR group with 6 patients ( 1 man, 5 women, average age 44,6 years, all of them without any treatment), and C (control) group with 8 healthy participants (3 men, 5 women, average age 36,8 years). All patients took part in project "Healthy life with diabetes". They were informed about experiment and related risks, and consents were obtained from all participants. The study protocol was approved by Bioethics Committee of the Medical University of Silesia -KNR/0022/KB1/82/II/15/16.

\section{Patient qualification}

In this study we considered four clinical criteria for patients' qualification: presence of type 2 diabetes based on level of HbA1c (normal range 4\%-5,6\%); BMI level (healthy weight:18,5-24,99 kg/m2; overweight: $25 \leq \mathrm{kg} /$ $\mathrm{m} 2$ ); lipid parameters disorders (healthy levels: $\mathrm{LDL} \leq 100$ $\mathrm{mg} / \mathrm{dL} ; \mathrm{HDL} \geq 60 \mathrm{mg} / \mathrm{dL} ; \mathrm{TG} \leq 150 \mathrm{mg} / \mathrm{dL}$ ) and insulin resistance based on HOMA-IR (optimal range $\leq 1.9$ ).

The metabolic qualification was performed by assessment of carbohydrate and lipid disturbances in the blood serum: glucose level and HbA1c (glycated haemoglobin), the level of insulin resistance determined by HOMA - IR method and lipid profile (LDL cholesterol, HDL cholesterol, TG - triglycerides). Additional measurements included anthropometric parameters with body mass index (BMI), body composition using bioimpedance analysis and the assessment of fatty liver using ultrasound imaging methods. Characteristics of metabolic parameters has been shown in Table 1. Other parameters relating to general condition of the body: renal (creatinine) and liver indicators (AlAt, AspAT), thyroid hormones (TSH), blood morphology, and inflammation marker (CRP) were assessed and were in normal level (data not shown).

Patients from IR group and $C$ patients did not suffer from type 2 diabetes and they did not use any medicines in comparison to T2D group. However, some patients were characterized by BMI level over $25 \mathrm{~kg} / \mathrm{m}^{2}$ and dyslipidemia (higher than healthy level of low-density lipoproteins- LDL and triglycerides). Liver fat in ultrasound imaging was also observed. Moreover, some of examined healthy volunteers had level above 1.9 of HOMA-IR.

Based on these findings, potentially healthy volunteers were classified into the IR group because they fulfill criteria of insulin resistance as a prediabetes state when blood glucose level is higher than normal but not high enough to be diagnosed as diabetes.

\section{Isolation of adipose derived stem cells}

Human mesenchymal stem cells were isolated from selected patients as was described (Bunnell et al. 2008; Cheng et al. 2011; Francis et al. 2010). Adipose tissue samples were washed with phosphate-buffered saline (PBS) containing antibiotics: penicillin/streptomycin (Lonza, Switzerland) and amphotericin B (Lonza, Switzerland). Then, adipose samples were minced and incubated in collagenase type I (Lonza, Switzerland) for $2 \mathrm{~h}$ at $37^{\circ} \mathrm{C}$ with shaking. The collagenase digestion reaction was stopped by adding DMEM medium with $10 \%$ fetal bovine serum. Stromal vascular fraction (SVF) was obtained by centrifuging at $1200 \mathrm{RPM}$ for $5 \mathrm{~min}$. The 
Table 1 Characteristics of metabolic parameters

\begin{tabular}{|c|c|c|c|c|}
\hline Parameters & Unit & $\begin{array}{l}\text { T2D group } \\
\text { (mean/median) }\end{array}$ & $\begin{array}{l}\text { IR group } \\
\text { (mean/median) }\end{array}$ & $\begin{array}{l}\text { Control group } \\
\text { (mean/median) }\end{array}$ \\
\hline Age & years & $45.3 / 44$ & $44.6 / 42$ & $36.8 / 35.5$ \\
\hline Sex & - & $3 \hat{0} 69$ & $10^{\lambda} 5 q$ & $3 \hat{0} 5 q$ \\
\hline BMI & $\mathrm{kg} / \mathrm{m} 2$ & $38.1 / 39$ & $24 / 25$ & $24 / 24$ \\
\hline Visceral index (bioimpedance) & - & $13.6 / 11.5$ & $6.3 / 5.5$ & $4.3 / 4$ \\
\hline Fasting plasma glucose & $\mathrm{mg} / \mathrm{dl}$ & $145 / 130$ & $79 / 80$ & $77 / 78$ \\
\hline $\mathrm{HbA1c}$ & $\%$ & $7.2 / 7.2$ & $5.2 / 5.3$ & $5.2 / 5.3$ \\
\hline HOMA-IR & - & $11.5 / 9.6$ & $2.3 / 2.6$ & $1.3 / 1.3$ \\
\hline HDL (high-density lipoprotein) & $\mathrm{mg} / \mathrm{dl}$ & $40 / 41$ & $69 / 73$ & $64 / 63$ \\
\hline LDL (low-density lipoprotein) & $\mathrm{mg} / \mathrm{dl}$ & $94 / 91$ & $125 / 130$ & $111 / 111$ \\
\hline TG (triglycerides) & $\mathrm{mg} / \mathrm{dl}$ & $183 / 152$ & $115 / 102$ & $83 / 81$ \\
\hline Liver fat in ultrasound imaging & - & present & absent/present & absent \\
\hline Serum Insulin & $\mu \mathrm{U} / \mathrm{ml}$ & $33 / 32.1$ & 11.6/13.7 & $6.9 / 6.7$ \\
\hline
\end{tabular}

upper oil fraction was discarded. The pellet was transferred into culture dishes, resuspended and incubated overnight for selection of adherent cells in DMEM medium (Dulbecco's Modified Eagle Medium, Lonza, Switzerland). Adherent cells were passaged when they reached 70\% confluence (Bunnell et al. 2008; Cheng et al. 2011).

\section{Cell culture conditions}

Normal human adipose derived stem cells (ADSCs) were routinely maintained in DMEM medium (Skubis et al. 2017; Sikora et al. 2019), supplemented with fetal bovine serum (FBS, EuroClone, Italy), amphotericin $\mathrm{B}$ and a penicillinstreptomycin mixture at $37^{\circ} \mathrm{C}$ in a $5 \% \mathrm{CO}_{2}$ incubator (Direct Heat $\mathrm{CO}_{2}$; Thermo Fisher Scientific, USA).

The culture medium was changed at intervals of 3 days. The experiment was performed on cells in the logarithmic phase of growth under condition of $\geq 98 \%$ viability assessed by trypan blue exclusion. Cells were assessed using the Olympus IX81 microscope (Olympus, Shinjuku, Tokyo, Japan) and DP70 camera (Olympus, Shinjuku, Tokyo, Japan) was used to photographic documentation. The ADSCs used for the experiment were at $2^{\text {nd }}$ passage, cultured in 6-well, 12-well and 96-well plates.

\section{Cell proliferation assays}

Adipose derived stem cells were plated at density of $3 \times$ $10^{3}$ per well in 96-well plates and incubated for $72 \mathrm{~h}$. After that WST-1 (Roche, Switzerland) and sulforodamine B (SRB, Sigma-Aldrich, USA) assays were done.

For WST-1 absorbance of dye was measured at a wavelength of $450 \mathrm{~nm}$ and for SRB assay absorbance of dye was measured at a wavelength of $590 \mathrm{~nm}$ in 96-well plate (Thermo Fisher Scientific, USA) using Microplate Reader Perkin Elmer Wallac Victor 2 (Perkin Elmer, USA). All the tests were performed in septuplicate.

\section{Flow cytometry analysis}

Human Mesenchymal Stem Cell Verification Flow Kit (FMC020, R\&D Systems, USA) was used for mesenchymal stem cells identification. Kit includes all of the antibodies required for assessing MSC marker expression according to the International Society Cell Therapy's definition of human MSCs and the appropriate isotype positive and negative controls. Kit contains conjugated antibodies for positive markers (CD73-CFS, CD90-APC, CD105-PerCP) and negative markers (CD45-PE, CD34PE, CD11b-PE, CD79A-PE, HLA-DR-PE). Cell fluorescence was measured immediately after staining (FACS Aria 2; Becton Dickinson), and data were analyzed using software (BD FACSDiva Software, Becton Dickinson). The results are expressed as counts per 10,000 events. The frequency of positive cells was measured as the percentile of gated cells in fluorescent channels with activities of the corresponding isotype controls.

\section{Osteoblast differentiation}

For these experiments, 20.000 cells per well were plated into 12-well cell culture plates. Second passage cells were used in the following studies. Cells were plated and grown until $75 \%$ confluent. Subsequently, the medium was replaced with fresh DMEM and 10\% FBS, $50 \mu \mathrm{M} \mathrm{L}$ ascorbic acid 2-phosphate, $10^{-7} \mathrm{M}$ dexamethasone and $10 \mathrm{mM} \beta$-glycerophosphate (Sigma-Aldrich, USA). Cells were cultured for 21 days. After that cells were collected and stored in $-20{ }^{\circ} \mathrm{C}$ until next analysis and Alizarin Red S (Sigma-Aldrich, St Louis, MO, USA) staining was made. Sample of every patient were cultured in triplicate.

\section{Alizarin red staining}

Alizarin red staining is used to evaluate calcium deposits by cultured cells. Alizarin red at concentration of 40 
$\mathrm{mM}$ was prepared in $\mathrm{dH}_{2} \mathrm{O}$ and the $\mathrm{pH}$ was adjusted to 4.1 using $10 \%$ ammonium hydroxide. For quantification of staining, $10 \%$ acetic acid (Sigma-Aldrich, USA) was added to each well for dissolving calcium deposits. The supernatants were read at $405 \mathrm{~nm}$ in 96-well plate using Microplate Reader Perkin Elmer Wallac Victor 2.

\section{Quantitative real-time polymerase chain reaction assay}

Total RNA was extracted from cells using a TRIzol reagent (Invitrogen, USA). RNA extracts were treated with DNase I (MBI Fermentas, Lithuania) according to the manufacturer's instructions. RNA concentration was determined using a GeneQuant II RNA/DNA spectrophotometer (Pharmacia Biotech, UK).

Expression assessment of MKI67 and phospho-histone H3 (pH 3), ALP, RUNX2, BGLAP, SPP1 was carried out using a real time RT-qPCR technique with SYBR Green chemistry (SYBR Green Quantitect RT-PCR Kit, Qiagen, Germany) and Opticon ${ }^{\text {tw }}$ DNA Engine Continuous Fluorescence detector (MJ Research, USA) as described previously (Strzalka et al. 2008). All samples were tested in triplicate. $\beta$-actin was included as an endogenous positive control (housekeeping gene) of amplification and integrity of RNA extracts. Oligonucleotide primers (MKI67, ALP, RUNX2, BGLAP, SPP1) were obtain in Sigma-Aldrich company (Sigma-Aldrich, USA). The primers for amplification of $p H 3$ mRNA were designed using Primer Express 1.0, ABI PRISM (Applied Biosystems, USA) (Orchel et al. 2004). Each reaction was completed using melting curve analysis to confirm the specificity of amplification and the absence of primer dimers.

\section{Statistical analysis}

Statistical analyses were performed using Statistica 13.0 software. Values were expressed as median value (Me) with the $25^{\text {th }}$ and $75^{\text {th }}$ quartiles, and minimum and maximum for non-normally distributed data and for normally distributed variables are presented as mean and standard deviation. Different groups were compared using Kruskal-Wallis test for non-normally distributed data and ANOVA with post hoc Tuckey for normally distributed. The level of significance was set at $p<0.05$ for all statistical tests.

\section{Results}

Analysis of viability in the ADSC - WST-1 and SRB assays Cell viability was determined using the WST-1 proliferation assay, which is based on mitochondrial activity. Results obtained from the assay showed that the proliferation of cells from patients from the IR group was the highest (one-way ANOVA, post hoc Tukey, $p<$ $0,05)$. We observed a statistically significant increase in viability in cells from IR patients as compared to control cells $(p=0.0001)$. The proliferation of cells from the
T2D group was significantly lower than for the control cells $(p=0.0083)$. Moreover, a statistically significant decrease in viability was observed in cells from T2D patients compared to the IR group ( $p=0.0001)$.

The cell viability was also assessed by measuring the total protein content in cells (SRB test) in all examined groups (Kruskal-Wallis test, $p<0.05$ ). Cells from IR patients showed higher viability compared to the control cells $(p<0.0001)$ and a statistically significant higher viability than T2D cells $(p=0.0036)$. Moreover, there was no difference in cells from T2D patients compared to control cells (Fig. 1).

\section{Analysis of $M K 167$ and $p H 3$}

An analysis of the mRNA levels of the MKI67 and $p H 3$ genes allowed for an estimation of the cell proliferation. The MKI67 mRNA level was significantly higher in cells from T2D patients compared to the control $(p=0.0001)$ and IR cells $(p=0.0002)$. Similarly, the expression of $p H$ 3 was higher in cells from the T2D group compared to the control $(p=0.0001)$ and IR cells $(p=0.0002)$ (Fig. 2).

\section{Analysis of ADSC phenotype}

We performed a fluorescence-activated cell sorting (FACS) analysis, which showed the expression of cell surface CD73, CD90, and CD105-specific markers of MSCs to assess the phenotype of the examined cells. The percentage of marker-positive cells was different in the examined groups. CD73 expression was significantly higher in the T2D group compared to the control cells $(p=0.0129)$. The expression of CD90 was statistically significantly lower in T2D cells compared to control $(p=0.0002)$ and IR cells $(p=0.001)$. The analysis of CD105 also showed statistically lower expression in T2D cells compared to the control $(p=0.0007)$ and IR cells $(p=0.011)$. Moreover, the expression of CD105 was significantly lower in T2D cells compared to IR cells ( $p=$ 0.001) (Fig. 3). Also, morphology of cells from T2D, IR and $\mathrm{C}$ groups were analyzed and we did not observe any differences in shape of cells (Fig. 4).

\section{Analysis of the osteogenesis potential of ADSCs}

We observed a higher expression of RUNX2 in cells in T2D group compared to the control cells $(p=0.0038)$ and cells from IR patients $(p=0.0104)$. Moreover, similar results were proved in the expression levels of SPP1 and $A L P$, Expression was higher in T2D cells in comparison to the control (SPP1, $p=0.0001 ; A L P, p<0.0001)$ and to cells from patients with insulin resistance $(S P P 1, p<$ $0.0001 ; A L P, p=0.0263)$. Interestingly, the analysis showed that the expression of BGLAP was lower in the IR group versus the control $(p=0.0263)$. Additionally, the mRNA level of BGLAP was higher in T2D cells compared to the control $(p<0.0001)$. There was also a 

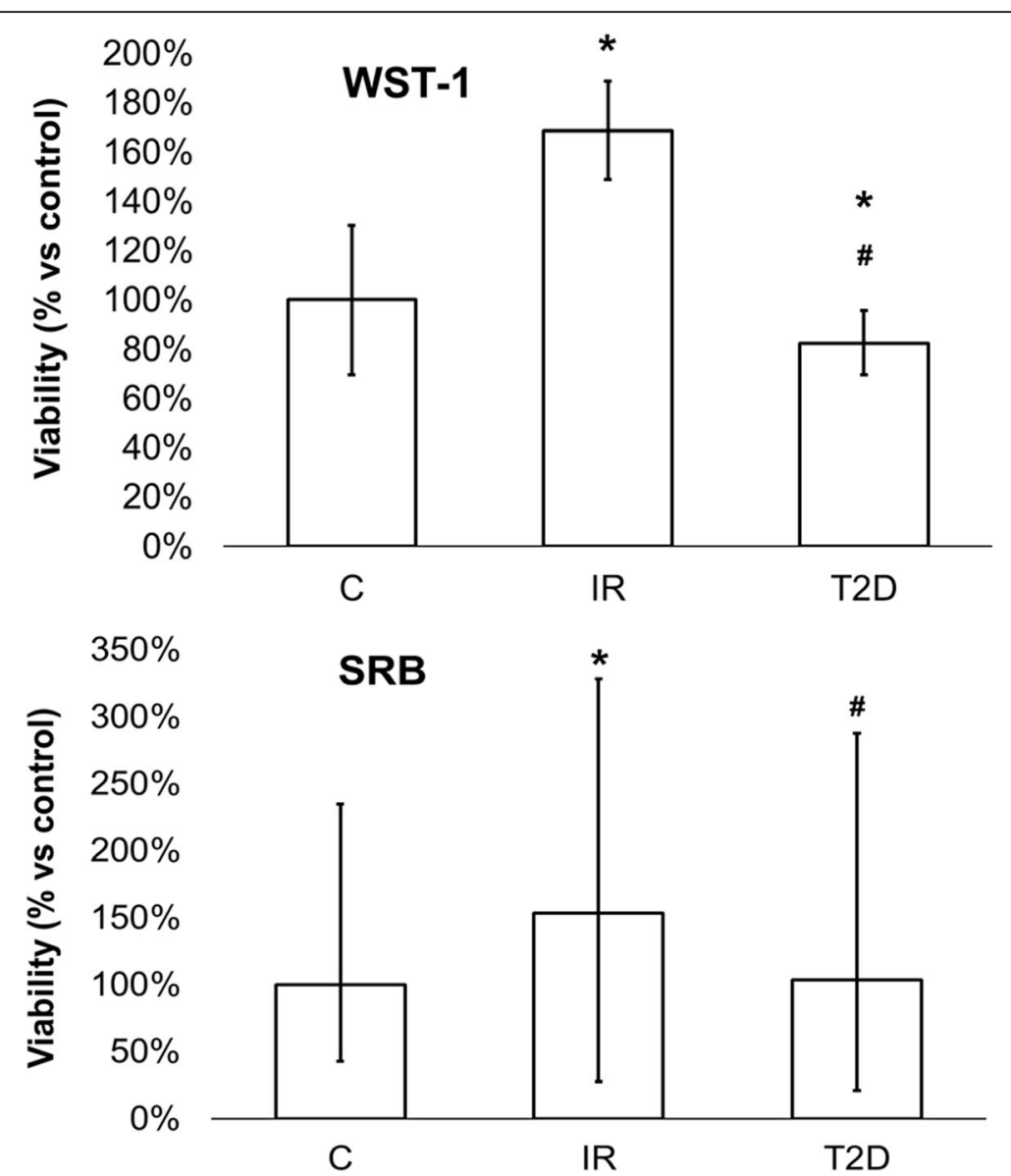

Fig. 1 Cells viability based on the measurement of mitochondrial oxidative (WST-1 assay) in cells and total protein content (SRB assay) from patients with insulin-resistance (IR), type 2 diabetes (T2D) compared to control cells (C) from healthy people. The bars represent the means \pm standard deviation (SD), ANOVA with the Tukey post hoc test (WST-1 assay) and the (Me) with the $25^{\text {th }}$ and $75^{\text {th }}$ quartiles and the minimum and maximum; the Kruskal Wallis test with post hoc (SRB assay), ${ }^{*} p<0.05$ vs. C, \#p $<0.05$ vs. IR

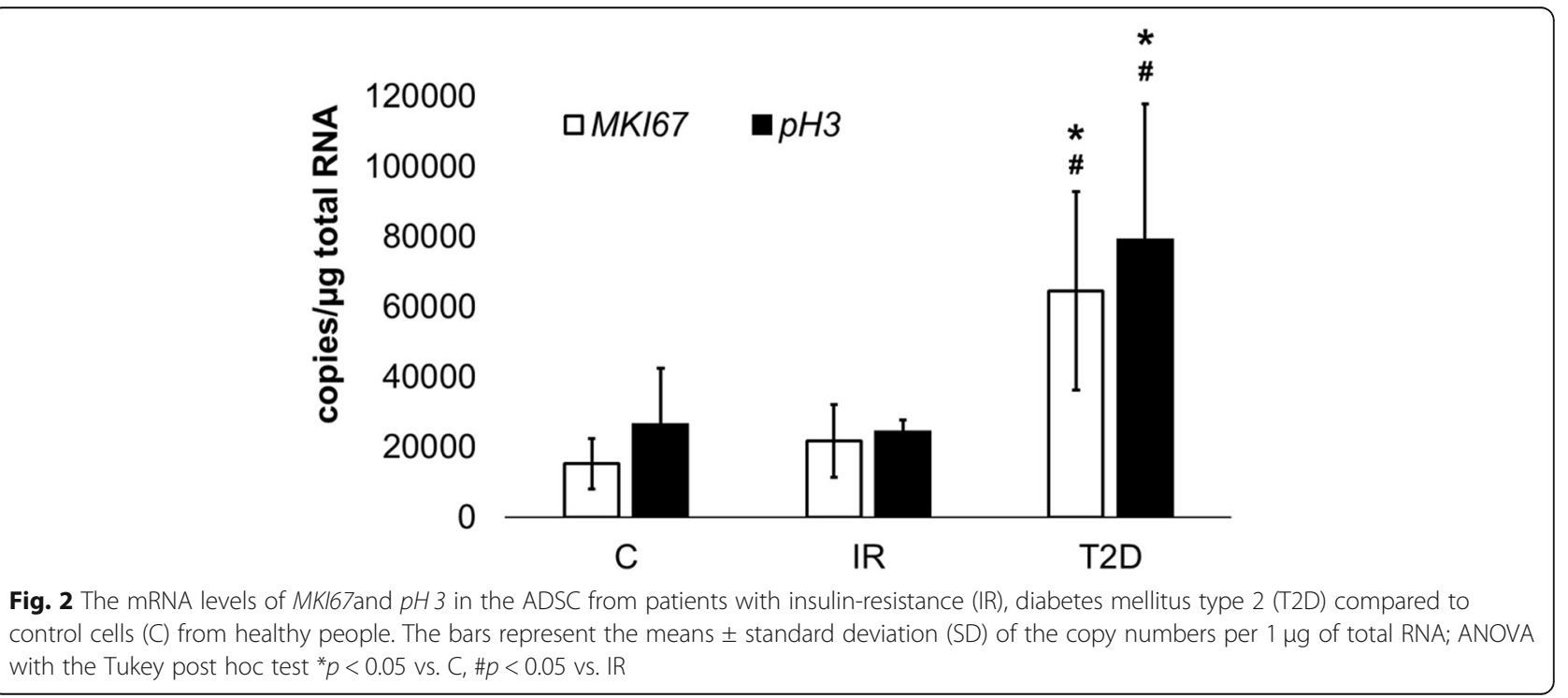




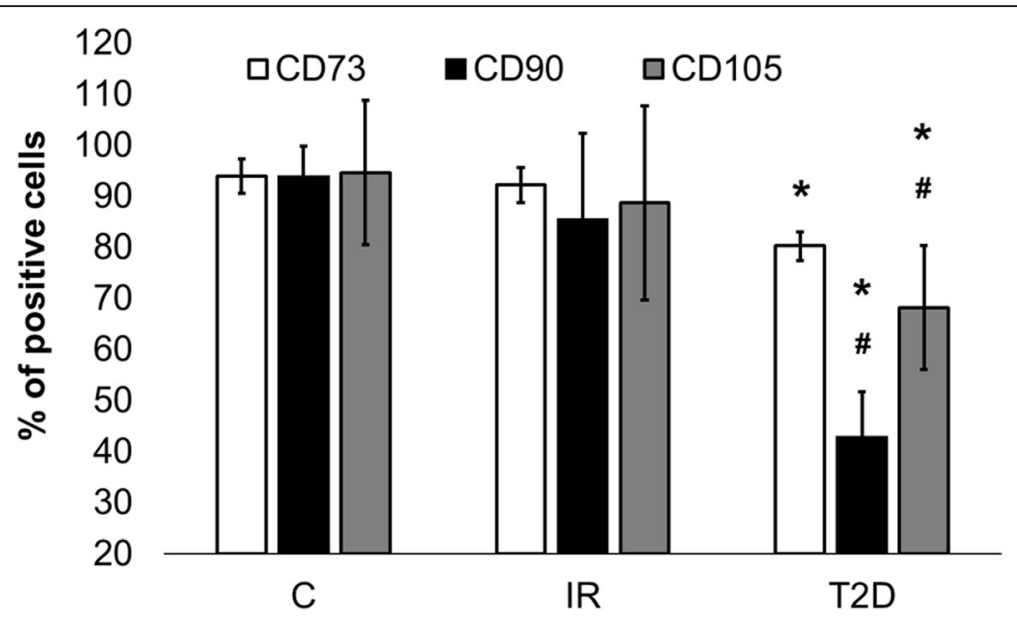

Fig. 3 The percentage of positive cells for CD73, CD90 and CD105 markers in cells from patients with insulin-resistance (IR), type 2 diabetes (T2D) compared to control cells (C). The bars represent the means \pm standard deviation (SD); ANOVA with the Tukey post hoc test ${ }^{*} p<0.05$ vs. $C$, \#p $<$ 0.05 vs. IR

statistically significant higher expression of $B G L A P$ in cells from the T2D group versus the IR group $(p<$ 0.0001) (Fig. 5).

For osteogenesis, the presence of extracellular calcium was confirmed by Alizarin red staining. Calcium deposition was also analyzed after 21 days of culturing (Fig. 6). The calcium level was measured and expressed as $\mathrm{mM}$ of calcium. Samples were evaluated in triplicate. The analysis showed a statistically significant higher level of calcium in cells from T2D patients versus the control $(p=0.0001)$ and IR cells $(p=0.0028)$.

\section{Discussion}

Type 2 diabetes is a metabolic disorder that begins with insulin resistance and is associated with a constantly high glucose level. The result of this abnormal insulin activity is a state of hyperglycemia, which contributes to a number of dysfunctions in body and tissues: the excessive production of reactive oxygen species in mitochondria (Jiao et al. 2015); the increase in protein oxidation, lipid peroxidation, and nucleic acid damage, all leading to cell damage (Jiao et al. 2015; Zabłocka and Janusz 2008); and chronic, difficult-to-soothe inflammation (Jiao et al. 2015). Additionally, nephropathy-impaired wound healing as well as irregularities in the bone and skeletal system have also been observed (Cheng et al. 2016).

Osteogenesis is a multistage process that is controlled in vivo by many molecular pathways. MSCs from adipose tissue can differentiate into mesodermal cells, such as osteoblasts, and they can be used in bone regeneration. Unfortunately, ADSCs differ between patients, especially due to a variety of diseases or drug use (Efimenko et al. 2015; Minteer et al. 2015), which can inhibit the efficacy of autologous cell therapy. Clinical studies have revealed that patients with T2D have more complications with bone healing (Dufrane 2017; Liang et al. 2014). Some studies have shown that diabetes

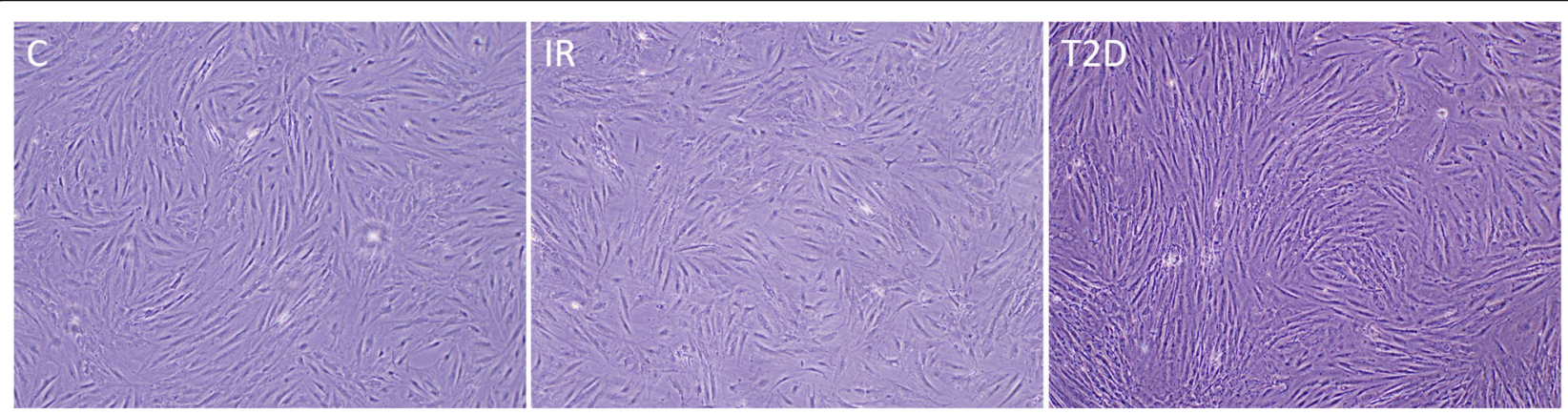

Fig. 4 The morphology of ADSCs from patients with insulin-resistance (IR), type 2 diabetes (T2D) compared to control cells (C) 


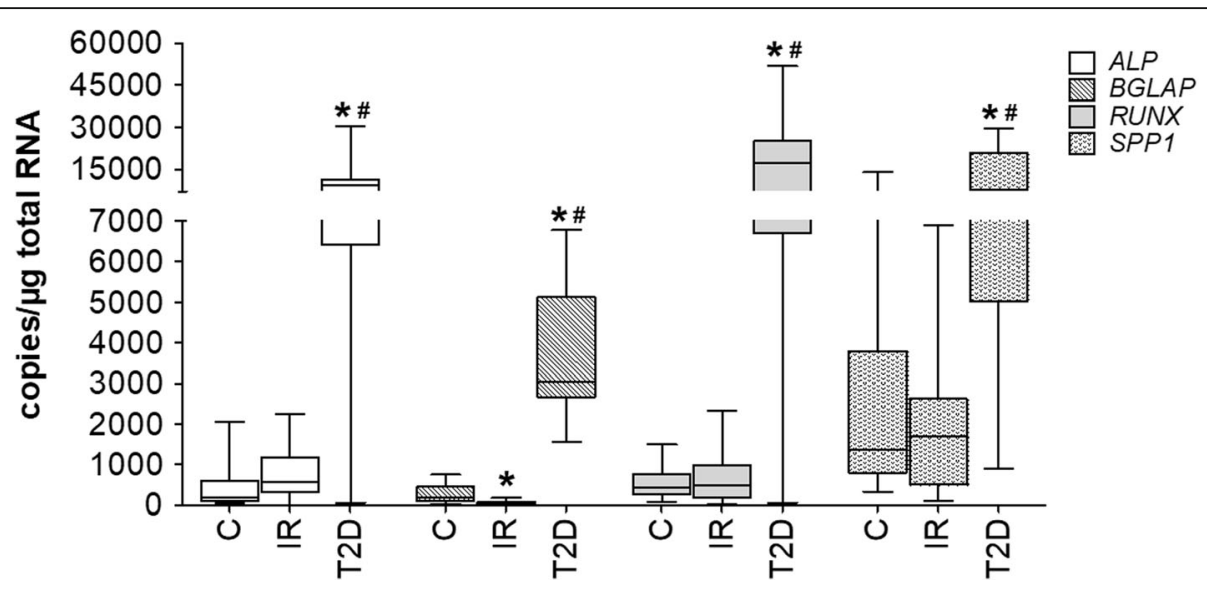

Fig. 5 The mRNA levels of BGLAP, ALP, SPP1, RUNX2 in the ADSC from patients with insulin-resistance (IR), type 2 diabetes (T2D) compared to control cells (C) from healthy people after osteoblast differentiation. The bars represent the (Me) with the $25^{\text {th }}$ and $75^{\text {th }}$ quartiles and the minimum and maximum of the copy numbers per $1 \mu \mathrm{g}$ of total RNA; the Kruskal Wallis test with post hoc, ${ }^{*} p<0.05$ vs. C, $\# p<0.05$ vs. IR

alters the proliferation and activity of ADSCs (Marycz et al. 2016; Nawrocka et al. 2017; Serena et al. 2016).

In our research, we examined the proliferation of ADSCs from patients with IR and T2D. We analyzed the cell proliferation based on a mitochondrial activity assay and a total protein content measurement. It was confirmed by both assays that ADSCs from patients with IR demonstrated a higher proliferation compared to the control. This proliferation may result from IR, hormonal imbalances, and, consequently, constant higher levels of glucose (Ko et al. 2015; Lee et al. 2018). Moreover, only the WST-1 assay showed a decreased proliferation in T2D cells in comparison to IR cells. Our results also revealed the upregulation of MKI67 and $p H 3$, molecular markers of proliferation (Orchel et al. 2004; Thompson et al. 2015), in cells from the T2D group compared to cells from the control and IR patients. It has been proven that medicines such metformin influences many molecular pathways (Hur and Lee 2015; Viollet et al.

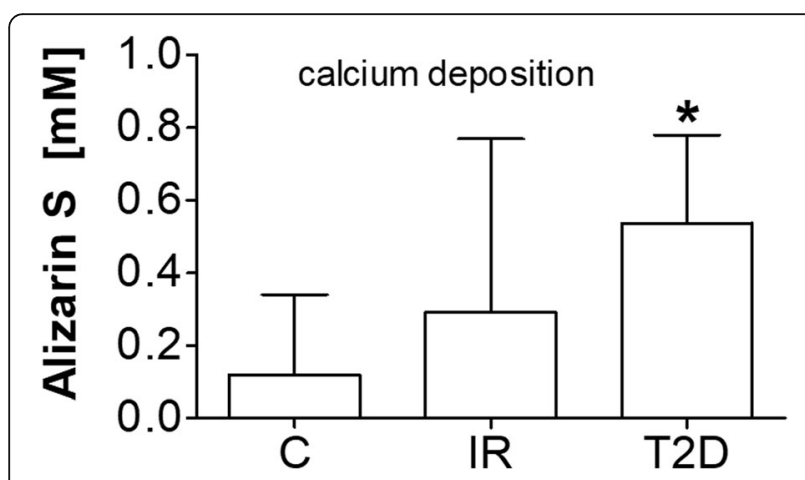

Fig. 6 Alizarin red S concentration $[\mathrm{mM}]$ in examined groups; insulin-resistance (IR), type 2 diabetes (T2D) compared to control cells (C); ANOVA with the Tukey post hoc test ${ }^{*} p<0.05$ vs. C
2012), and it is likely that differences in the cytotoxicity assay results and the mRNA profiles may be related to the medicines taken by T2D patients, but further studies are required to confirm this observation.

However, the upregulation of MKI67 and $p H 3$ mRNA expression may be due to the fact that these cells were cultured in standard conditions in vitro with different glucose level in comparison to the conditions which were present in patients organisms. The reason of the upregulation of the proliferation markers gene expression may be associated with the potential regeneration of ADSC derived from diabetic patients. However, this effect on the metabolic level assessed by WST-1 and SRB assays could be yet undetectable, because the transcription and changes at the genome level precede significantly the phenotype changes where the metabolic activity and the protein production are included.

Our finding showed that cells from T2D patients have a lower expression of membrane proteins characteristic for stem cells: CD73, CD90, and CD105. The results did not show a cellular change for IR patients at the protein level.

Our study showed that the differentiation of ADSCs from T2D patients into osteoblasts was stronger than in cells from the control group or IR patients. Both type 1 and type 2 diabetes are associated with an increased risk of bone fractures. In the course of the disease, abnormal microarchitecture and quality of bone are seen, as well as irregularities in new bone formation (Räkel et al. 2008). Hyperglycemia resulting in decreased expression of the genes encoding for markers of osteoblast formation also leads to an increased expression of proinflammatory cytokines. This all contributes to a reduction in osteoblast activity and differentiation and induces their apoptosis. Another mechanism of hyperglycemia is the promotion 
of adipogenesis in MSCs using high glucose concentrations, which is connected to the inhibition of MSCs differentiation into osteoblasts by changing the differentiation towards that of adipocytes, instead (Yan and Li 2013). It was suggested that the high glucose levels inhibited the differentiation of osteoblasts and osteoblast precursors (Jiao et al. 2015; Li et al. 2010). Moreover, a high concentration of glucose leads to the previously mentioned formation of advanced glycation end products (AGEs) and reactive oxygen species (ROS) over-production. The final AGEs stimulate osteoclast formation and induce the osteoclastogenic process and inhibit the differentiation of osteoblasts by the decreased expression of alkaline phosphatase and collagen $1 \alpha 1$. There is also evidence that AGEs induce apoptosis of osteoblasts (Jiao et al. 2015). ROS stimulate the differentiation and survival of osteoclasts and promote apoptosis of osteoblasts. The long-term effect of oxidative stress is the reduction of bone mass (Jiao et al. 2015). Increased levels of TGF- $\beta$ in the serum of people with diabetes leads to the reduced activity of alkaline phosphatase, which is responsible for providing the necessary phosphate in the matrix mineralization. The result is a decrease in matrix mineralization and abnormal bone formation (Ehnert et al. 2015). Inflammatory factors induce and maintain the regulation of these processes through the bone resorption by osteoclasts in the process of osteoclastogenesis. The inflammatory process in diabetes can reduce the number of osteoblasts via the induction of apoptosis. In diabetes, an increased expression of pro-apoptotic genes, including an increased $\mathrm{Bax} / \mathrm{Bcl}-2$ ratio, was reported, which corresponds with increasing of apoptosis (Jiao et al. 2015).

We observed a higher potential for osteogenesis based on the mRNA level of BGLAP, SPP1, ALP, and SPP1 in patients with T2D. The same results were also observed in the Alizarin red staining for calcium deposition. We did not observe a significant change in IR patients in comparison to the control, suggesting that hyperglycemia is not a main differentiation factor.

Many studies suggest that metabolic diseases like diabetes inhibit the activity and differentiation ability of stem cells. Less is known about the influence of diabetes pharmacotherapy on stem cell function, especially in human models. Most studies have focused on animal models, though there are many factors that change the activity of cells in humans. Apart from diabetes treatment, other factors might have influenced these results, including comorbidities, age, and length of disease. Such factors cannot be assessed in animal models.

\section{Conclusion}

We have concluded that type 2 diabetes changes the activity of stem cells, and insulin resistance influences on the proliferation of ADSCs.

\begin{abstract}
Abbreviations
ADSCs : Adipose-derived stem cells; AGEs: Advanced glycation end products; AlAt: Alanine aminotransferase; ALP: Alkaline phosphatase; ANOVA : Analysis of variance; APC: Allophycocyanin; AspAT: Aspartate aminotransferase; BMI: Body mass index; BGLAP : Osteocalcin; CD105 : Endoglin; CD73 : Cluster of differentiation 73/ecto-5'-nucleotidase; CD90 : Cluster differentiation 90/ Thy-1 antigen; CD45: Protein tyrosine phosphatase/ receptor type C; CD34: Hematopoietic Progenitor Cell Antigen CD34; CD11b: Integrin subunit alpha M/ITGAM; CD79A: B-cell antigen receptor complex-associated protein alpha chain; CFS: Carboxyfluorescein; CRP: C-reactive protein; dH2O : Distilled water; DMEM : Dulbecco's Modified Eagle Medium; DNA: Deoxyribonucleic acid; FACS: Fluorescence-activated cell sorting; FBS : Fetal bovine serum; HbA1c: Glycated haemoglobin; HDL: High-density lipoprotein; HLA-

DR: Human Leukocyte Antigen - DR isotype; HOMA - IR: Homeostatic model assessment for insulin resistance; IR: Insulin resistance; LDL: Low-density lipoprotein; mE: Median; MKI67: Marker of proliferation Ki-67; mRNA

: Messenger ribonucleic acid; MSC : Mesenchymal stem cells; PBS : Phosphate buffered saline; PE: Phycoerythrin; PerCP: Peridinin-chlorophyll-protein; $\mathrm{pH}$ 3: Phospho histone h3; RNA: Ribonucleic acid; ROS: Reactive oxygen species; RTq PCR : Quantitative real-time polymerase chain reaction; RUNX2 : Runtrelated transcription factor 2; SPP1: Osteopontin; SRB : Sulforhodamine B; SVF : Stromal vascular fraction; T1D: Type 1 diabetes; T2D: Type 2 diabetes; TG: Triglyceride; TGF- $\beta$ : Tansforming growth factor beta; TSH: Thyrotropic hormone; WST-1: (4-[3-(4-lodophenyl)-2-(4-nitrophenyl)-2H-5-tetrazolio]-1,3benzene disulfonate)
\end{abstract}

\section{Acknowledgments}

We would like to express our deepest appreciation to dr hab. Monika PaulSamojedny for help with cytometric analysis and invaluable contribution to this work.

\section{Authors' contributions}

Aleksandra Skubis-Sikora, Bartosz Sikora and Agnieszka Witkowska conceived and designed the experiments; Bartosz Sikora, Aleksandra Skubis-Sikora performed the experiments; Aleksandra Skubis-Sikora and Joanna Gola analyzed the data; Urszula Mazurek contributed reagents/materials/analysis tools; Aleksandra Skubis-Sikora, Joanna Gola, Agnieszka Witkowska and Bartosz Sikora wrote the paper. The author(s) read and approved the final manuscript.

\section{Funding}

Authors kindly acknowledge the support from Medical University of Silesia in Katowice (Upper Silesia, Poland) grants no. KNW-2-37/D/7/N, KNW-2-38/D/7/ N.

\section{Availability of data and materials \\ Not applicable.}

Ethics approval and consent to participate

The study protocol was approved by Bioethics Committee of the Medical University of Silesia -KNR/0022/KB1/82/II/15/16.

\section{Consent for publication}

Not applicable.

\section{Competing interests}

The authors declare that there is no conflict of interest regarding the publication of this article.

\section{Author details}

${ }^{1}$ Department of Cytophysiology, Chair of Histology and Embryology, Faculty of Medical Sciences in Katowice, Medical University of Silesia in Katowice, ul. Medyków 18, C2/108, 40-752 Katowice, Poland. ²Fundacja Zdrowego Życia, ul. Kotlarza 6, 40-139 Katowice, Poland. ${ }^{3}$ Józef Tyszkiewicz Higher School in Bielsko-Biała, ul, Nadbrzeżna 12, 43-300 Bielsko-Biała, Poland. ${ }^{4}$ Department of Molecular Biology, Chair of Molecular Biology, Faculty of Pharmaceutical 
Sciences in Sosnowiec, Medical University of Silesia in Katowice, Katowice, Poland.

Received: 31 January 2020 Accepted: 19 June 2020

Published online: 02 July 2020

\section{References}

Amin M, Suksomboon N. Pharmacotherapy of type 2 diabetes mellitus: an update on drug-drug interactions. Drug Saf. 2014;37:903-19.

Bunnell B, Flaat M, Gagliardi C, Patel B, Ripoll C. Adipose-derived stem cells: isolation, expansion and differentiation? Methods. 2008:45:115-20.

Cheng K-H, Kuo T-L, Kuo K-K, Hsiao C-C. Human adipose-derived stem cells: isolation, characterization and current application in regeneration medicine. Genomic Med, Biomarkers Health Sci. 2011;3:53-62.

Cheng N-C, Hsieh T-Y, Lai H-S, Young T-H. High glucose-induced reactive oxygen species generation promotes stemness in human adipose-derived stem cells. Cytotherapy. 2016;18:371-83.

Dufrane D. Impact of age on human adipose stem cells for bone tissue engineering. Cell Transplant. 2017;26:1496-504

Dzhoyashvili NA, et al. Disturbed angiogenic activity of adipose-derived stromal cells obtained from patients with coronary artery disease and diabetes mellitus type 2. J Transl Med. 2014;12:337.

Efimenko AY, Kochegura TN, Akopyan ZA, Parfyonova YV. Autologous stem cell therapy: how aging and chronic diseases affect stem and progenitor cells. BioResearch Open Access. 2015;4:26-38.

Ehnert $\mathrm{S}$, et al. Factors circulating in the blood of type 2 diabetes mellitus patients affect osteoblast maturation - description of a novel in vitro model. Exp Cell Res. 2015;332:247-58

Evert $A B$, et al. Nutrition therapy recommendations for the Management of Adults with Diabetes. Diabetes Care. 2013:36:3821-42.

Francis MP, Sachs PC, Elmore LW, Holt SE. Isolating adipose-derived mesenchymal stem cells from lipoaspirate blood and saline fraction. Organogenesis. 2010;6:11-4.

Hur KY, Lee M-S. New mechanisms of metformin action: focusing on mitochondria and the gut. J Diabetes Investig. 2015;6:600-9.

Jiao H, Xiao E, Graves DT. Diabetes and its effect on bone and fracture healing. Curr Osteoporos Rep. 2015;13:327-35.

$\mathrm{Ko} \mathrm{Kl}$, et al. Diabetes reduces mesenchymal stem cells in fracture healing through a TNFa-mediated mechanism. Diabetologia. 2015;58:633-42.

Lee J-M, et al. Obesity alters the long-term fitness of the hematopoietic stem cell compartment through modulation of Gfil expression. J Exp Med. 2018;215: 627-44.

Li H, Jiang L-S, Dai L-Y. High glucose potentiates collagen synthesis and bone morphogenetic protein-2-induced early osteoblast gene expression in rat spinal ligament cells. Endocrinology. 2010;151:63-74.

Liang $L$, et al. Adipose-derived stem cells combined with inorganic bovine bone in Calvarial bone healing in rats with type 2 diabetes. J Periodontol. 2014;85:601-9.

Marycz K, Kornicka K, Basinska K, Czyrek A. Equine metabolic syndrome affects viability, senescence, and stress factors of equine adipose-derived Mesenchymal stromal stem cells: new insight into EqASCs isolated from EMS horses in the context of their aging. Oxidative Med Cell Longev. 2016;2016:1-17.

Minteer DM, et al. Analysis of type II diabetes mellitus adipose-derived stem cells for tissue engineering applications. J tissue Eng. 2015;6:2041731415579215.

Nawrocka D, Kornicka K, Szydlarska J, Marycz K. Basic fibroblast growth factor inhibits apoptosis and promotes proliferation of adipose-derived mesenchymal stromal cells isolated from patients with type 2 diabetes by reducing cellular oxidative stress. Oxidative Med Cell Longev. 2017;2017:1-22.

O'Hagan C, De Vito G, Boreham CAG. Exercise prescription in the treatment of type 2 diabetes mellitus: current practices, existing guidelines and future directions. Sports Med. 2013;43:39-49.

Orchel J, Slowinski J, Mazurek U, Wilczok T. H3 mRNA level as a new proliferative marker in astrocytomas. Biochim Biophys Acta (BBA) - Mol Basis Dis. 2004; 1689:42-6.

Räkel A, Sheehy O, Rahme E, LeLorier J. Osteoporosis among patients with type 1 and type 2 diabetes. Diabetes Metab. 2008:34:193-205.

Serena $C_{\text {, et }}$ al. Obesity and type 2 diabetes alters the immune properties of human adipose derived stem cells: obesity changes the immune properties of stem cells. Stem Cells. 2016:34:2559-73.

Sikora B, Skubis-Sikora A, Kimsa-Furdzik M, et al. Adipose-derived stem cells undergo differentiation after co-culture with porcine limbal epithelial stem cells. Stem Cell Res. 2019;41:101609. https://doi.org/10.1016/j.scr.2019.101609.
Simpson C, et al. The effects of diabetes medications on post-operative long bone fracture healing. Eur J Orthop Surg Traumatol. 2015;25:1239-43.

Skubis A, Gola J, Sikora B, et al. Impact of antibiotics on the proliferation and differentiation of human adipose-derived mesenchymal stem cells. Int J Mol Sci. 2017;18(12):2522. https://doi.org/10.3390/ijms18122522.

Stankov K, Benc D, Draskovic D. Genetic and epigenetic factors in etiology of diabetes mellitus type 1. Pediatrics. 2013;132:1112-22.

Strzalka B, et al. Quantitative analysis of transforming growth factor [beta] isoforms mRNA in the human corneal epithelium. Folia Biol. 2008;54:46.

Thompson MD, et al. Lack of effect of metformin on mammary carcinogenesis in nondiabetic rat and mouse models. Cancer Prev Res. 2015:8:231-9.

Viollet B, et al. Cellular and molecular mechanisms of metformin: an overview. Clin Sci. 2012;122:253-70.

Yan W, Li X. Impact of diabetes and its treatments on skeletal diseases. Front Med. 2013;7:81-90

Zabłocka A, Janusz M. Dwa oblicza wolnych rodników tlenowych the two faces of reactive oxygen species. Postepy Hig Med Dosw(online). 2008;62:118-24.

\section{Publisher's Note}

Springer Nature remains neutral with regard to jurisdictional claims in published maps and institutional affiliations.

Ready to submit your research? Choose BMC and benefit from:

- fast, convenient online submission

- thorough peer review by experienced researchers in your field

- rapid publication on acceptance

- support for research data, including large and complex data types

- gold Open Access which fosters wider collaboration and increased citations

- maximum visibility for your research: over $100 \mathrm{M}$ website views per year

At BMC, research is always in progress.

Learn more biomedcentral.com/submissions 